\title{
Early Maturation of the Linguistic Dorsal Pathway in Human Infants
}

\author{
François Leroy, ${ }^{1,2,3}$ Hervé Glasel, ${ }^{1,2,3}$ Jessica Dubois, ${ }^{1,2,3}$ Lucie Hertz-Pannier, ${ }^{3,4,5,6}$ Bertrand Thirion, ${ }^{3,7}$ \\ Jean-François Mangin, ${ }^{3,8}$ and Ghislaine Dehaene-Lambertz ${ }^{1,2,3}$ \\ ${ }^{1}$ INSERM, U992, Neurospin, 91191 Gif/Yvette, France, ${ }^{2}$ Université Paris 11, 91405 Orsay, France, ${ }^{3}$ IFR49, Neurospin, 91191 Gif/Yvette, France, ${ }^{4}$ INSERM, \\ U663, 75015 Paris, France, ${ }^{5}$ Université Paris 5, 75015 Paris, France, ${ }^{6}$ Commissariat à l'Energie Atomique (CEA), Laboratoire de Recherche Biomédicale, \\ NeuroSpin, 91191 Gif-sur-Yvette, France, ${ }^{7}$ INRIA, Saclay-Ile-de-France, Parietal Project team, 91191 Gif-sur-Yvette, France, and ${ }^{8} \mathrm{CEA}$, Laboratoire de \\ Neuroimagerie Assistée par Ordinateur, NeuroSpin, 91191 Gif-sur-Yvette, France
}

Human infants, unlike even closely related primates, exhibit a remarkable capacity for language learning. Yet how the underlying anatomical network matures remains largely unknown. The classical view is that of a largely immature brain comprising only a few islands of maturity in primary cortices. This view has favored a description of learning based on bottom-up algorithms and has tended to discard the role of frontal regions, which were assumed to be barely functional early on. Here, using an index based on the normalized T2-weighted magnetic resonance signal, we have quantified maturation within the linguistic network in fourteen 1- to 4-month-old infants. Our results show first that the ventral superior temporal sulcus (STS), and not the inferior frontal area, is the less mature perisylvian region. A significant difference of maturation in the STS favoring the right side is an early testimony of the distinctive left-right development of this structure observed during the whole life. Second, asymmetries of maturation in Broca's area were correlated with asymmetries in the posterior STS and in the parietal segment of the arcuate fasciculus, suggesting that an efficient frontotemporal dorsal pathway might provide infants with a phonological loop circuitry much earlier than expected.

\section{Introduction}

Speech processing in adults relies on distributed networks of the left-hemisphere frontal and temporal lobes. These distant areas are linked by two pathways: a dorsal suprasylvian pathway via the arcuate and the superior longitudinal fasciculus, and a ventral pathway running through the uncinate and the extreme capsule system (Anwander et al., 2007; Frey et al., 2008). How the development of these regions buttresses language acquisition in humans is still unknown. From universal predisposition at birth, infants rapidly converge on the features of their environmental language during the first year of life, first in the perception domain and later on in production. The mechanisms behind the adjustments to the native language are generally described as based on statistical analyses of the speech input within general auditory or speech-dedicated networks (Saffran et al., 1996; Maye et al., 2002), facilitated by the prosodic organization of speech (Johnson and Jusczyk, 2001). Although not anatomically specified, these models can be considered as bottom-up models, expected to mainly involve auditory regions in the superior temporal areas. This view was supported by the scant data published

Received Aug. 8, 2010; revised 0ct. 25, 2010; accepted Nov. 16, 2010.

This research was financially supported by the McDonnell Foundation, La Fondation Motrice, fondation de France, and Agence Nationale pour la Recherche. J.D. was funded by Ecole des Neurosciences de Paris-lle de France and Fyssen Foundation. We thank T. Brunet from Ravier-Touzard Company for designing a baby bouncer chair specifically adapted to the head coil, F. Brunelle for his support, F. Rousseau for sharing his software.

Correspondence should be addressed to Ghislaine Dehaene-Lambertz, INSERM, U992, Neurospin, Bat 145, point courrier 146, 91191 Gif//vette, France. E-mail: ghislaine.dehaene@cea.fr.

DOI:10.1523/JNEUROSCI.4141-10.2011

Copyright $\odot 2011$ the authors $\quad 0270-6474 / 11 / 311500-07 \$ 15.00 / 0$ on early human brain development. Postmortem studies have emphasized the delayed and protracted development of the prefrontal areas in humans relative to visual and auditory cortices (Flechsig, 1920; Yakovlev and Lecours, 1967; Kinney et al., 1988; Huttenlocher and Dabholkar, 1997). Metabolic studies confirmed that frontal regions have a lower glucose metabolism until 6-8 months, when it gradually increases first in the lateral prefrontal regions, then in the medial part. Metabolism needs are evenly distributed only after the end of the first year (Chugani et al., 1987).

Recent functional imaging studies have challenged this picture. Three-month-old infants, and even neonates, recruit areas beyond the temporal lobes: the inferior and dorsolateral frontal regions when engaged in a speech task (DehaeneLambertz et al., 2002, 2006a; Gervain et al., 2008; Bristow et al., 2009) and the anterior prefrontal cortex when social cues are present (Grossmann et al., 2008; Dehaene-Lambertz et al., 2010). The role of frontal areas in language learning might thus have been neglected.

Our goal in this paper is thus to obtain a more precise description of the maturation of the perisylvian areas thanks to magnetic resonance imaging (MRI) spatial resolution. We used the MR T2-weighted (T2w) signal itself as a direct window on the cortical changes induced by maturation processes. T2 $\mathrm{w}$ signal decreases with brain maturation because it is affected by changes in the ratio of free to bound water produced by the proliferation of membranes (growth of axons and dendrites, proliferation and differentiation of glia cells), by the increase of hydrophobic proteolipids constituting the myelin (Barkovich, 2000), and by the 


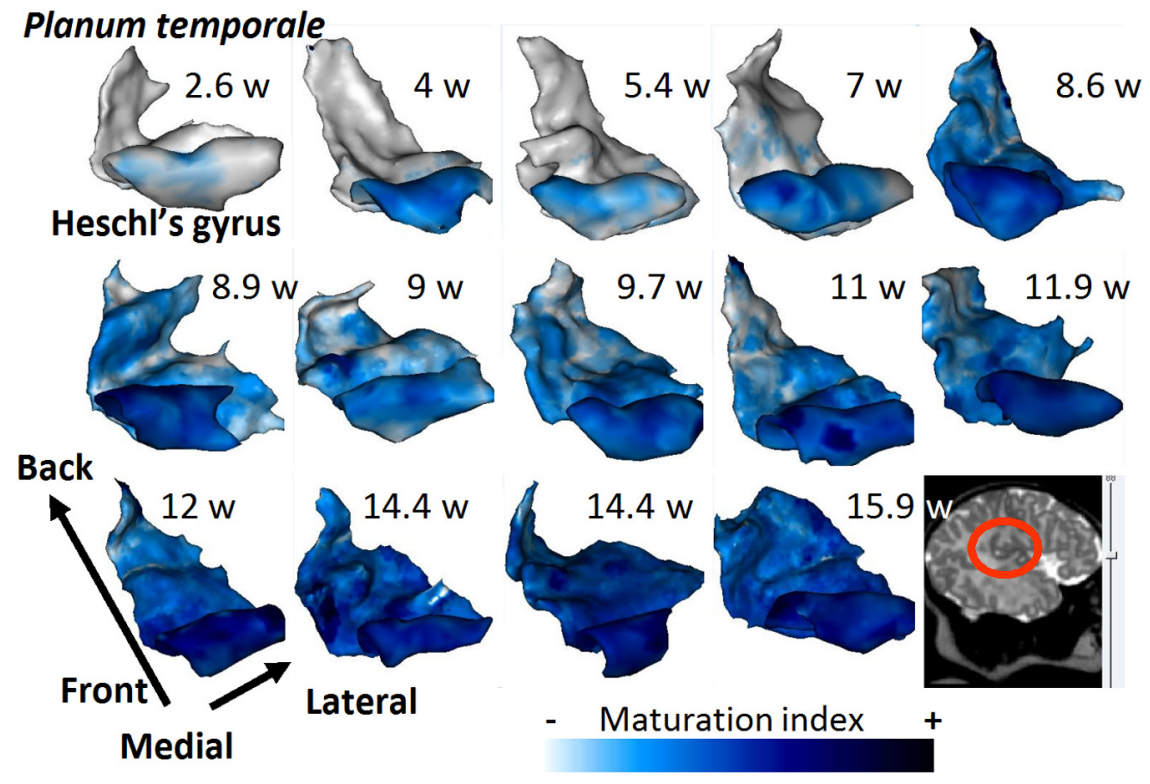

Figure 1. Illustration of the effect of age on the maturation index. The maturation index of the adjacent cortex is projected on 3-D meshes of the left planum temporale and Heschl's gyrus in the 14 infants ordered by age. As infants grow older, maturation progresses from the medial to the lateral part and from front to back.

deposits of ferritin (Fukunaga et al., 2010). The heterogeneous aspect of T2w images during the first year of life has been linked to the differential maturational tempo of the different brain areas, and rough milestones, useful in clinical practice, have been established (Barkovich, 2000). We propose here that a normalized index based on this signal can be used to precisely quantify gray matter maturation in the infant brain.

\section{Materials and Methods}

\section{Subjects}

Fourteen healthy full-term infants ( 9 boys, 5 girls, age range: $2.6-16.3$ weeks) were included after their parents gave written informed consent. The infants were spontaneously asleep during MR imaging. The study was approved by the regional ethical committee for biomedical research.

\section{Data acquisition}

MR acquisitions were performed with a T2-weighted fast spin-echo sequence $(\mathrm{TE} / \mathrm{TR}=120 / 5500 \mathrm{~ms}$ ) on a $1.5 \mathrm{~T}$ MRI system (Signa LX, GEMS), using a birdcage head coil. Images covering the whole brain were acquired along the axial, sagittal, and coronal orientations for each infant $\left(0.8 \times 0.8 \times 2 \mathrm{~mm}^{3}\right.$ in each orientation, field of view: $20 \mathrm{~cm}$, raw matrix: $192 \times 192$ interpolated to $256 \times 256)$. During the same run, diffusion tensor images were also acquired in 13 on 14 infants $\left(b=700 \mathrm{~s} / \mathrm{mm}^{2}\right.$, $\mathrm{TE} / \mathrm{TR}=89.6 \mathrm{~ms} / 13.8 \mathrm{~s}, 14-30$ encoding orientations of the diffusion gradient, spatial resolution interpolated to $0.94 \times 0.94 \times 2.5 \mathrm{~mm}^{3}$ at reconstruction).

\section{Data postprocessing}

For each infant, one high-resolution image $\left(1 \times 1 \times 1 \mathrm{~mm}^{3}\right)$ was reconstructed from the three-orientation images (Rousseau et al., 2006). Because the contrast between white and gray matter is weak, heterogeneous across brain areas, and rapidly changing during the first months of life, automatic brain segmentation procedures are prone to errors. To obtain exact measures on well delineated structures, H.G. manually drew on both sides the superior temporal sulcus (STS), the sulcibordering Broca's area (sylvian rami, inferior precentral and inferior frontal sulci), those bordering the supramarginal gyrus, Heschl's gyrus, the planum temporale, and the central sulcus as a mature reference structure. Sulci were preferred to gyri because of their clearer boundaries and shape, which facilitate realignment between infants and between hemispheres.

\section{Statistical analyses}

We built a maturation index based on the normalized gray matter signal. First, a sphere of $1 \mathrm{~mm}$ radius was rolled along each drawn structure to recover the minimum $\mathrm{T} 2 \mathrm{w}$ signal within the sphere at each structure coordinate. Because gray matter tissue is the darkest signal in T2w images of immature brains, this minimum is expected to be relatively free of partial volume effects. Second, we used the CSF as a signal-intensity reference across infants. The CSF composition is stable and produces a strong signal, which is highly distinguishable from the dark gray matter. Furthermore, CSF close to our structures of interest is similarly affected by the low-frequency spatial variations of the signal due to radio frequency inhomogeneities (Vovk et al., 2007). We detected peripheral CSF using hysteresis threshold and removed partial volume effects from neighboring tissues using strong morphological erosion ( $1.5 \mathrm{~mm}$ radius). Finally, we computed the histogram of the eroded CSF regions and rejected the lowest signal values to get a symmetrical histogram. The final CSF mask was made of all remaining voxels. The CSF signal was then averaged within a $15 \mathrm{~mm}$ radius around each structure. For large structures (STS, central sulcus, inferior frontal-precentral sulcus, and Heschl's gyrus-planum temporale), the CSF signal was averaged at each coordinate over the intersection of the CSF mask and a tube around the structure centered at the given coordinate. The tube length ranges from $8 \mathrm{~mm}$ to $16 \mathrm{~mm}$ and $32 \mathrm{~mm}$ for Broca's sulci, the central sulcus, and the superior temporal sulcus, respectively. The range of the tube length results from a tradeoff between the number of voxels needed for a proper estimate of the CSF mean value (favoring a large tube) and the spatial extent of the distribution of the voxels to match the local signal bias (favoring a small tube). We could not obtain enough CSF voxels in the vicinity of three sulci in three different subjects (two STS and one central sulcus). These three sulci and their contralateral counterparts were therefore excluded from the study. Finally, we defined a maturation index as $1-S_{\text {cortex }} / S_{\mathrm{CSF}}$, for each flank of the sulci and each hemisphere (13 regions by two hemispheres). This index was expected to increase with age, as the gray matter signal decreases, whereas the CSF signal remains constant (Fig. 1). The values measured along the structures were averaged to get the cortical signal over the whole structure on each side. For large structures, we defined a maturation profile along their main axis (see Fig. 3). The profile was normalized by the square root of the hemispherical white matter envelope surface in each infant to take into account the brain growth during this time period.

\section{DTI postprocessing}

These data have already been published (Dubois et al., 2006, 2009). The arcuate and uncinate fasciculi, but not the extreme fasciculus, were reconstructed by tractography for these publications. We used here the fractional anisotropy (FA) values measured in these tracts from the 13 infants common in the present study and these DTI studies.

Validation of the index. We first checked that our index was indeed sensitive to maturation by examining how it correlated with age in each structure (linear or quadratic relationship). To demonstrate its spatial sensitivity, indexes computed on the two flanks of the same sulcus were compared with a two-tailed paired $t$ test.

Clustering model. We then examined how the different regions of interest clustered. A model selection approach was applied on a probabilistic clustering model to the cortical regions of interest where each region is characterized by its maturation index across subjects. Clustering was performed through a Gaussian mixture model, where the best-fitting model is kept across 100 random initializations. Model selection was 
performed using cross-validation. For each possible value of the number $K$ of components in the mixture model, the density of the maturation indexes is learned from $(n-1)$ brain regions, and this log-likelihood of the index from the remaining region is computed. The value of $K$ that yields the highest cross-validated log-likelihood is selected as the best one, and a final clustering of the brain regions is obtained with a mixture model that has the optimal number of components. The cross-validation procedure ensures that the selection is unbiased, even with a few numbers of observations. By contrast, standard penalization-based criteria (AIC, BIC) are valid only asymptotically, and typically overestimate $K$.

We run the model selection on structures from both hemispheres. Because indexes were missing for three sulci in three different infants, we applied the model selection to the remaining 11 subjects, as well as to all subjects using interpolated data when the maturation index was missing.

Asymmetry analyses. For each measure, we computed an asymmetry coefficient defined as $2 \times($ Right - Left $) /($ Right + Left $)$, and we tested whether it was significantly different from zero using two-tailed $t$ tests. For the long sulci, we applied permutation tests over the $t$ variable to determine the significant asymmetric segment. The most asymmetrical segment was estimated by applying 5000 permutation tests over the Student's $t$ variable. The paired $t$ score of the asymmetry coefficient was computed at each sulcal point using a sliding window, and the segment with the maximum $t$ score was identified. Then, the $t$ score distribution was estimated through $2 n$ random flips of right and left profiles across the $n$ subjects. Finally, the $t$ values obtained in the real data were compared to this distribution to estimate the $p$ value of the asymmetry in the given segment. We first detected asymmetrical segments using a 8 -mm-wide sliding window and then increased the window size to get its full spatial extent.

Correlation analyses between variables. Finally, we analyzed the relation between maturation asymmetries in the frontal and temporal areas. To reduce the number of comparisons, our analyses were driven by the adults' literature. Recent adults' data have shown a clear division between the ventral and dorsal pathway (Lerch et al., 2006; Anwander et al., 2007; Frey et al., 2008). Area 44, which is located in the pars opercularis along the anterior bank of the precentral sulcus, is preferentially connected to the posterior temporal regions through the arcuate, whereas area 45 , located anteriorly in the pars triangularis, is preferentially connected to anterior STS through the uncinate and the extreme capsule system. We thus first computed a regression analysis between asymmetry coefficients of the Broca's side of the precentral sulcus on one hand (area 44), and the planum temporale and the dorsal and ventral flanks of the STS on the other hand. We expected from the literature significant correlation with posterior segments of these structures. Second, we similarly examined whether maturation in the other and more anterior sulci of Broca's area (inferior frontal sulcus, Broca's rami) were related to the ventral and dorsal STS, expecting significant correlations in this case with more anterior segments of STS. Finally we examined whether there was a correlation between gray matter and white matter maturation indexes. We thus computed regressions between FA asymmetry of the uncinate fasciculus and of the parietal and temporal segments of the arcuate fasciculus on one hand and our index of asymmetry in Broca's sulci and in the STS on the other hand. Note that we used the asymmetry coefficients rather than the maturation indices themselves for two reasons. First, asymmetries might be related to the development of the linguistic function, and thus the time lag between left and right maturation would be a more sensitive variable than the pure values of maturation in each side. Second, because of the important differences of maturation across structures and subjects, a normalized variable, such as asymmetry coefficients, is less variable than the original indexes and thus should be more sensitive.

For each analysis, a linear regression of age was first performed on the variables to remove any age confound, and the residuals of that regression were entered in the regression analyses instead of the original values. When a significant correlation was observed between two structures, we used permutation tests on the original values to identify the gray matter segment showing the highest correlation and to estimate its corrected $p$ value.

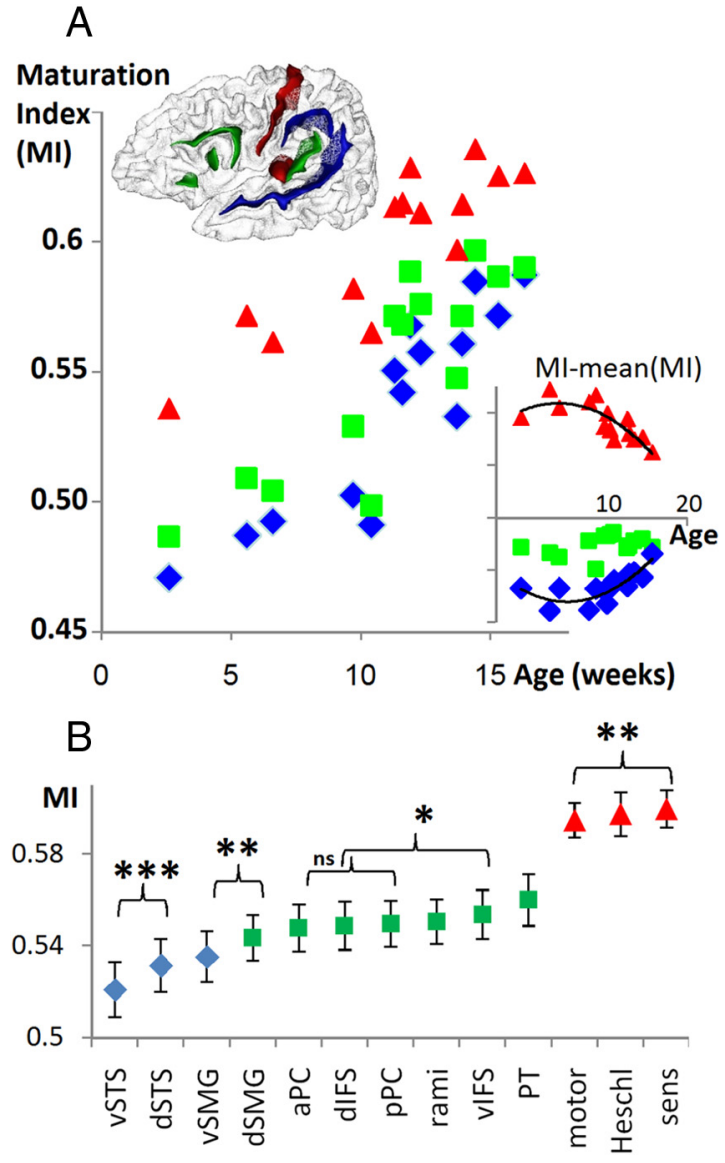

Figure 2. A, Perisylvian structures grouped by maturation level. Scatter plot of the maturation index (MI) according to age for each maturational group. The studied structures, colored depending on the maturation group they belong to, are presented on a 3-D 2-month-old infant's brain mesh. Primary cortices (central sulcus and Heschl's gyrus) belong to the most mature group (in red). The inferior frontal sulci and the planum temporale (PT) cluster together in an intermediate group (in green). The STS and the supramarginal gyrus (SMG) are the most immature structures (in blue). The scatter plot of the differences between maturation indexes and the mean is presented enclosed in the main plot to illustrate the different maturation rates of the three groups. The difference follows a quadratic function of age for groups 1 (inverted $U$ curve $R^{2}=0.63, p=0.04$ ) and 3 (U curve $R^{2}=0.52, p=0.03$ ). $\boldsymbol{B}$, Scatter plot of the different structures ordered by MI. Each colored symbol corresponds to the maturation group each region belongs to. Embraces indicate the two flanks of the same sulcus $\left({ }^{*} p<0.05\right.$, ${ }^{* *} p<0.01$, $\left.{ }^{* * *} p<0.001\right)$. v, Ventral; d, dorsal; a, anterior; $p$, posterior; $P C$, precentral; IFS, inferior frontal sulcus.

\section{Results}

Because of the difficulties of brain-tissue segmentation at this age, our analyses were limited to manually drawn structures (i.e., the main perisylvian sulci plus the central sulcus as a reference). We expected that a valid maturation index should change with age and discriminate at least between primary and associative cortices. Correlation with age was indeed high in each region (mean $R^{2}=0.88$ : 0.82-0.94 with a linear model). The highest index, indicating maturity, was observed for the somatosensory cortex, followed by Heschl's gyrus and the motor cortex, and then the associative cortices (Fig. 2; for more details, see supplemental Fig. 1 , available at www.jneurosci.org as supplemental material). The significant difference between the two banks of the central sulcus (somatosensory more mature than motor: $F_{(1,12)}=9.5, p=$ $0.009)$ provided an internal validation that our measures were anatomically precise and sensitive to different maturation rates even in neighboring areas. Finally, we observed a catching up of 


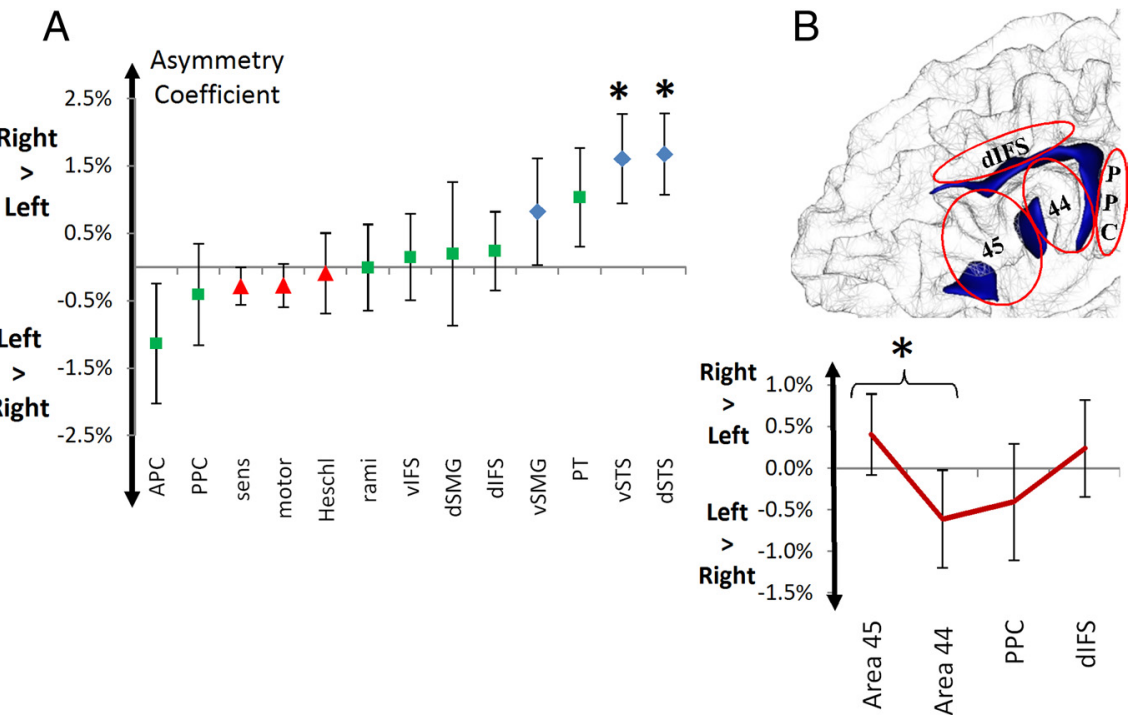

Figure 3. Maturation asymmetries. $A$, Scatter plot of the asymmetry coefficients ordered by size. The colored symbols correspond to the maturation groups as defined in the main text and Figure 2 . See Figure 2 for structure abbreviations. ${ }^{*}$ corresponds to a $p$ value $<0.5$ in the $t$ test comparison of left versus rightindexes for each structure (dorsal STS: $t_{(11)}=2.7, p=0.021$, ventral STS: $t_{(11)}=2.2, p=0.047, \mathrm{APC}: t_{(13)}=1.38$, $p=0.19) \cdot \boldsymbol{B}$, Asymmetry coefficient in the inferior frontal region. Brodmann area 44 was significantly more asymmetric than area 45.
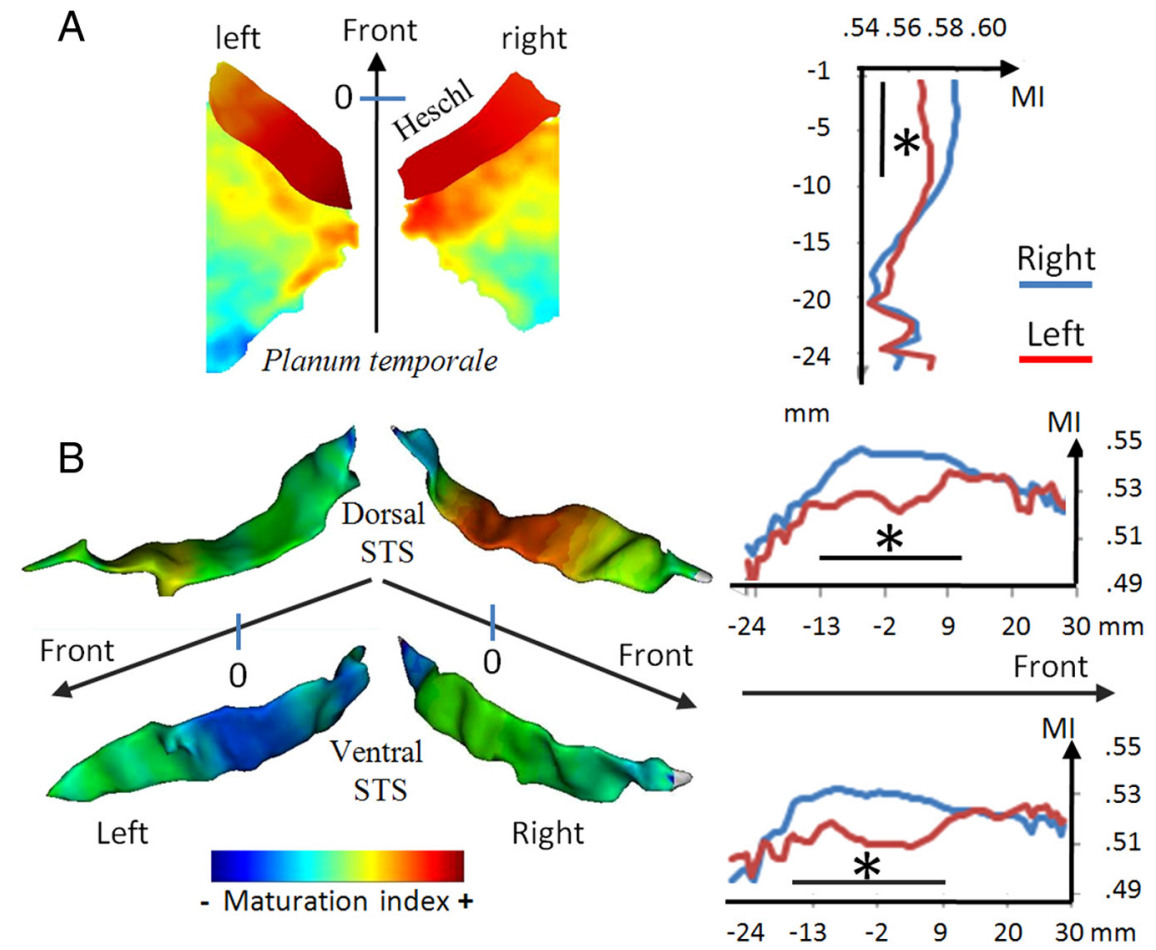

Figure 4. Maturation asymmetries in the temporal areas. $A$, Two-dimensional projection on a regular grid of the mean value of the maturation index (MI) across infants in Heschl's gyrus and planum temporale. Note the larger left planum and the anteriorposterior and medial-lateral gradient of maturation in both Heschl's gyrus and the planum. To keep color information in Heschl's gyrus, which is a more mature structure, the color map has a logarithmic scale. The right column presents the MI measured along the planum anterior-posterior axis. $\boldsymbol{B}$, Mean maturation index of the left and right STS projected on an individual 3-D mesh of the sulcus. The right column presents the MI measured along the anterior-posterior axis of both sulcal banks. Black horizontal lines show the spatial extent of the significant asymmetry measured in each point through permutations. A right lead is present for these three structures. Note also the dorsal-ventral gradient of maturation (planum $>$ dorsal STS $>$ ventral STS).

associative regions with primary cortices after 10 weeks, captured by a quadratic function of age $\left(R^{2}=0.68, p=0.002\right)$. This decrease of the difference between cortices with age highlighted the different maturation tempo of these regions.
Our results, however, go beyond the classical division between primary and associative cortices. Using a model selection approach, we identified three maturation groups (log-likelihood for one-, two-, three-, and four-class models using the dataset comprising the 11 infants with a complete set of sulci: $22.7 / 30.3 / 31 / 30$ and $29.3 / 39 / 40.4 / 39$ when the 14 infants are considered): (1) The most mature group included, as expected, the primary cortices: Heschl's gyrus and both banks of the central sulcus. (2) An intermediate maturation group consisted of the planum temporale and all inferior frontal sulci. Within this group, the planum temporale was significantly more mature than the frontal sulci (paired $t$ test, $p$ values $<$ 0.02 ), whose indexes did not differ. (3) The most immature group comprised the cortices bordering the STS and the supramarginal gyrus (Fig. 2). Its dorsal bank was included with the intermediate group on the left and was either classified with the intermediate or the least mature group on the right depending on the considered dataset. This region is thus very close to the boundary between the two groups. The most immature structure within perisylvian areas was the ventral bank of the STS, which lagged significantly behind all other regions, and in particular behind its dorsal bank $\left(F_{(1,11)}=59.8, p<0.001\right)$. However, the difference of maturation between the STS and the frontal areas decreased as a quadratic function of age $\left(R^{2}=0.48, p=0.026\right)$, revealing a catching up with the frontal areas during the studied time period.

\section{Maturation asymmetries}

Asymmetry is a characteristic of the linguistic network. We thus examine in each structure whether the left and right perisylvian regions had a similar maturational calendar (Fig. 3). A robust and unexpected rightward bias was observed for the temporal structures (right $>$ left global index: dorsal STS: $t_{(11)}=2.7, p=$ 0.021 , ventral STS: $t_{(11)}=2.2, p=0.047$, two-tailed paired $t$ test) (Fig. 4). The asymmetric segment was $24 \mathrm{~mm}$ long and localized at the base of Heschl's gyrus and of the planum temporale (dorsal STS: $t=4.09$; corrected $p=0.015$; ventral STS: $t=3.44$; corrected $p=0.037$ ) (Fig. 4). The most anterior part of the planum along Heschl's gyrus ( $9 \mathrm{~mm}$ ) had a rightward coefficient $(t=3.25$; corrected $p=0.029)$, whereas Heschl's gyrus itself was not asymmetric $\left(t_{(13)}<1\right)$. The left dorsal STS was catching up with the right during the studied time period as indicated by a significant linear decrease of the asymmetry with age $\left(R^{2}=\right.$ 
$0.39, p=0.03)$. No other structure had a significant asymmetrical maturation. The structure with the most leftward coefficient was Broca's bank of the precentral sulcus, but this left lead was not significant $(p=0.19)$.

Because of their theoretical interest, we further analyzed asymmetries in Broca's region. Areas 44 and 45 constituting Broca's area are approximately located over the pars opercularis (area 44) and the pars triangularis (area 45). A rough estimation of their maturation can be obtained by averaging the maturation indexes of their bordering sulci (for area 44: the anterior bank of the precentral, the posterior bank of the ascending ramus, and the posterior half of the ventral bank of the inferior frontal sulcus; for area 45: the anterior bank of the ascending ramus, the anterior ramus, and the anterior half of the ventral bank of the inferior frontal sulcus). Area 44 was significantly more asymmetric toward the left than area $45\left(t_{(13)}=3.46, p=0.04\right)$. A developmental gradient in asymmetry beginning in the pars opercularis and extending progressively anteriorly toward area 45 and posteriorly to the precentral sulcus is compatible with the two published postmortem studies. The dendrite length of layer 5 pyramidal neurons shows an earlier shift for a left lead in Broca's area relative to the premotor cortex [Simonds and Scheibel (1989), their Table 5]. Amunts et al. (1995) reported that asymmetry in gray matter indexes increases with age in area 45 , while it was steadily established in area 44 even in the youngest infants ( $\sim 4$ months of age in their study). However, these results are weak and need further studies to be verified.

\section{Correlation between maturation indices}

To examine whether there was a relation between the development of frontal and temporal areas, we computed regression analyses between the asymmetry coefficients in the frontal and temporal areas. To remove any age confound, a linear regression of age was first performed, and the residuals of that regression were entered in the regression analyses instead of the original values. The only significant correlation was between Broca's bank of the precentral sulcus (area 44) and the ventral STS $\left(R^{2}=0.52\right.$, $p=0.008)$, especially its most posterior part bordered by the angular gyrus $\left(R^{2}=0.55, p=0.006\right)$ (Fig. 5). No other significant correlation was observed between other Broca's sulci and the temporal structures. We have previously reported that FA in the parietal sector of the arcuate was higher in the left than in the right, suggesting a faster left myelination (Dubois et al., 2009). Because of the tight connection between area 44 and the posterior temporal areas through this fasciculus (Anwander et al., 2007; Frey et al., 2008), we examined whether the maturation in the two regions revealed by the previous analysis was also correlated with the asymmetry coefficient in this white matter segment $\left(R^{2}=\right.$ $0.62, p=0.007$ for the posterior segment of the ventral STS and $R^{2}=0.32, p=0.053$ for the Broca's flank of the precentral sulcus). When correlations were examined along the whole STS and precentral/inferior frontal sulcus and this tract, the significant correlated segments were the same 8 -mm-long posterior segment in the ventral STS identified above (corrected $p=0.047$ ) and a 9-mm-long segment at the junction between the inferior frontal and the precentral structure (corrected $p=0.011$ ). By contrast, the same analyses performed on the uncinate fasciculus and on the temporal part of the arcuate did not reveal any significant correlation between FA asymmetries in these tracts and any frontal or temporal segment.

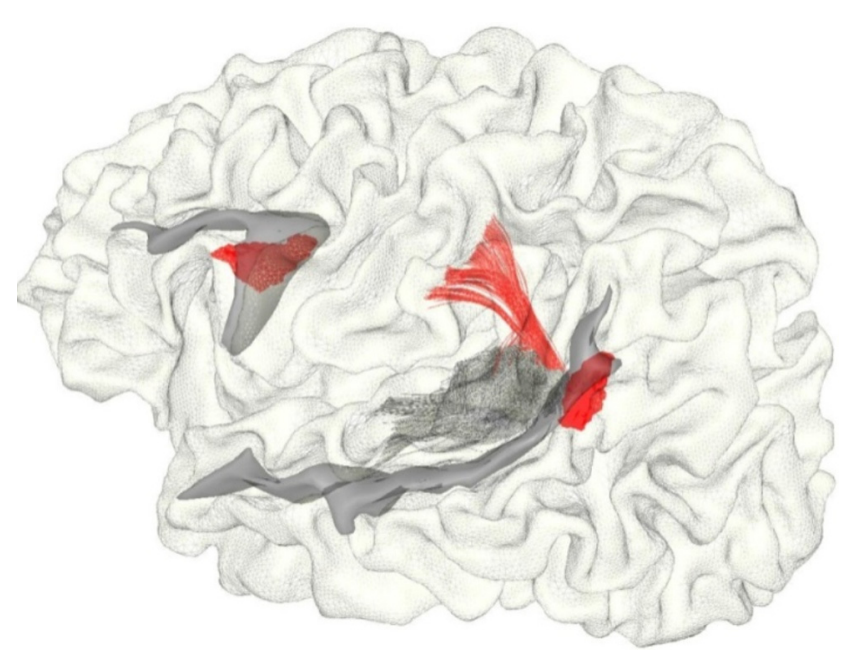

Figure 5. Correlations between white and gray matter maturation along the dorsal pathway. The STS, the precentral/inferior frontal sulci, and the arcuate fasciculus of an individual infant are presented on his brain mesh. The arcuate fasciculus is limited to the parietal and temporal sections easily identifiable in infants. The regions significantly correlated at a group level are marked in red and correspond to the parietal part of the arcuate, the ventral part of the posterior STS, and area 44 at the junction between the precentral and the inferior frontal sulcus.

\section{Discussion}

We first demonstrated that it was possible to use a normalized $\mathrm{T} 2 \mathrm{w}$ signal to quantify cortical maturation in precise brain regions. We reproduced the general rules proposed from postmortem studies (Yakovlev and Lecours, 1967; Kinney et al., 1988; Huttenlocher and Dabholkar, 1997), according to which primary cortices matured before associative cortices and sensory cortices before the motor cortex. Here, the highest index, indicating maturity, was observed for the somatosensory cortex, followed by Heschl's gyrus and the motor cortex, then associative cortices. Because MRI has better spatial resolution than positron emission tomography and single-photon emission computed tomography, it was also possible to distinguish different maturational levels or different maturation time courses even in close areas such as the two banks of the same sulci (sensory vs motor cortex, ventral vs dorsal bank of the STS) or between two neighboring areas (e.g., premotor vs motor areas) or along a sulcus (Figs. 1, 4).

It can, however, be argued that the differences we observed were not due to maturation but rather to differences in cellular organization between the different types of cortices. In adults, Sigalovsky et al. (2006) using the longitudinal relaxation time (R1) and Yoshiura et al. (2000) using T2 images observed subtle signal differences between Heschl's gyrus and other superior temporal regions related to a higher myelin content and cell density in the auditory koniocortex. The well known heterogeneous darkening of T2 images along the first year of life and the decrease of the difference between the STS and the frontal areas noted here during the studied time period (see also Fig. 2) demonstrate that the signal difference between areas is not constant and that maturation rather than cortical type is the main cause of the signal difference between areas at this age.

Our index can thus be used to estimate the relative maturation of the different perisylvian areas. We observed first a clear dorsalventral gradient of decreasing maturity along the superior temporal region (planum temporale $>$ dorsal STS $>$ ventral STS). Along the same axis, the blood oxygenation level-dependent response presents an increasing phase lag (Dehaene-Lambertz et al., 2006a). Anatomical and functional measures are thus congru- 
ent to split infant's temporal regions in subregions with different developmental and functional trajectories as described in monkeys (Kaas and Hackett, 2000) and human adults (DehaeneLambertz et al., 2006b).

Second, the left STS was significantly lagging behind the right. The observation of a STS asymmetry agrees with numerous studies at different ages pointing to the distinctive development of this structure. Between 12 and 14 weeks of gestation, several genes are asymmetrically expressed toward the right side in the human temporal region (Sun et al., 2005). Like a majority of sulci, the right STS appears 1 or 2 weeks earlier than the left in humans (Chi et al., 1977; Dubois et al., 2008). However, contrary to other sulci, it remains asymmetric, deeper on the right side, in infants (Hill et al., 2010; H., Glasel, F. Leroy, J. Dubois, L. Hertz-Pannier, J. Mangin, and G. Dehaene-Lambertz, unpublished work) and in adults (Ochiai et al., 2004; Van Essen, 2005). Gray-matter density also shows a distinctive pattern between left and right temporal regions along the entire lifespan (Sowell et al., 2003). Because the STS hosts several important functions besides language, such as social contact, biological motion perception, and audiovisual integration (Hein and Knight, 2008), further studies are needed to understand how the asymmetric pattern of development of this region may favor the development of the verbal and nonverbal human communication system.

Finally, our analyses crucially reveal that it is the superior temporal sulcus, and not the inferior frontal area, which is the most immature structure of the perisylvian network. Yet, the STS already displays high-level auditory properties at this age, such as detecting the repetition of a sentence (Dehaene-Lambertz et al., 2010) and detecting audiovisual congruence (Bristow et al., 2009). Thus, frontal maturation should be sufficient to sustain functional activity. This result bolsters the functional studies reporting that this area is active in infants when listening to speech (Dehaene-Lambertz et al., 2006a; Bristow et al., 2009). Moreover, correlation analyses revealed that temporal and frontal regions are not developing independently but show correlated interindividual variability over and above age-related changes. A similar result was reported in adolescents using cortical thickness as the variable (Lerch et al., 2006), and it was proposed that covariations in cortical thickness between distant brain areas reveal the architecture of the human brain (Chen et al., 2008). We observed here a correlation between regions belonging to the dorsal pathway (posterior STS, arcuate fasciculus, and area 44). This pathway is notably involved in the phonological loop underlying working memory, and thus might provide the neural substrate behind the enhancement of activity observed in Broca's area when syllables (Bristow et al., 2009) and sentences (Dehaene-Lambertz et al., 2006a) are repeated. It may also provide infants with an early interface between speech perception and production systems, through area 44, which is tightly connected with the motor and somatosensory areas. It may also be crucial in the turn-taking aspect of conversation as proposed by Scott et al. (2009), which develops early on between infant and parents. The expansion of the arcuate fasciculus, prominent in humans compared to other primates (Rilling et al., 2008), has been proposed as one important key in the emergence of language (Aboitiz et al., 2010). Note, however, that the absence of significant correlations between the more anterior part of the STS and anterior Broca's area does not mean that the ventral pathway is not functional at this age. Nevertheless, our results bring positive evidence of early efficient frontotemporal loops that should be acknowledged when explaining the first stages of language learning.

\section{References}

Aboitiz F, Aboitiz S, Garcia R (2010) The phonological loop. A key innovation in human evolution. Curr Anthropol 51:S55-S65.

Amunts K, Istomin V, Schleicher A, Zilles K (1995) Postnatal development of the human primary motor cortex: a quantitative cytoarchitectonic analysis. Anat Embryol (Berl) 192:557-571.

Anwander A, Tittgemeyer M, von Cramon DY, Friederici AD, Knösche TR (2007) Connectivity-based parcellation of Broca's area. Cereb Cortex $17: 816-825$.

Barkovich AJ (2000) Concepts of myelin and myelination in neuroradiology. AJNR Am J Neuroradiol 21:1099-1109.

Bristow D, Dehaene-Lambertz G, Mattout J, Soares C, Gliga T, Baillet S, Mangin JF (2009) Hearing faces: how the infant brain matches the face it sees with the speech it hears. J Cogn Neurosci 21:905-921.

Chen ZJ, He Y, Rosa-Neto P, Germann J, Evans AC (2008) Revealing modular architecture of human brain structural networks by using cortical thickness from MRI. Cereb Cortex 18:2374-2381.

Chi JG, Dooling EC, Gilles FH (1977) Gyral development of the human brain. Ann Neurol 1:86-93.

Chugani HT, Phelps ME, Mazziotta JC (1987) Positron emission tomography study of human brain functional development. Ann Neurol 22:487-497.

Dehaene-Lambertz G, Dehaene S, Hertz-Pannier L (2002) Functional neuroimaging of speech perception in infants. Science 298:2013-2015.

Dehaene-Lambertz G, Hertz-Pannier L, Dubois J, Mériaux S, Roche A, Sigman M, Dehaene S (2006a) Functional organization of perisylvian activation during presentation of sentences in preverbal infants. Proc Natl Acad Sci U S A 103:14240-14245.

Dehaene-Lambertz G, Dehaene S, Anton JL, Campagne A, Ciuciu P, Dehaene GP, Denghien I, Jobert A, Lebihan D, Sigman M, Pallier C, Poline JB (2006b) Functional segregation of cortical language areas by sentence repetition. Hum Brain Mapp 27:360-371.

Dehaene-Lambertz G, Montavont A, Jobert A, Allirol L, Dubois J, HertzPannier L, Dehaene S (2010) Language or music, mother or Mozart? Structural and environmental influences on infants' language networks. Brain Lang 114:53-65.

Dubois J, Hertz-Pannier L, Dehaene-Lambertz G, Cointepas Y, Le Bihan D (2006) Assessment of the early organization and maturation of infants' cerebral white matter fiber bundles: a feasibility study using quantitative diffusion tensor imaging and tractography. Neuroimage 30:1121-1132.

Dubois J, Benders M, Cachia A, Lazeyras F, Ha-Vinh Leuchter R, Sizonenko SV, Borradori-Tolsa C, Mangin JF, Hüppi PS (2008) Mapping the early cortical folding process in the preterm newborn brain. Cereb Cortex 18:1444-1454.

Dubois J, Hertz-Pannier L, Cachia A, Mangin JF, Le Bihan D, DehaeneLambertz G (2009) Structural asymmetries in the infant language and sensori-motor networks. Cereb Cortex 19:414-423.

Flechsig P (1920) Anatomie des menschlichen Gehirns und Rückenmarks auf myelogenetischer Grundlage. Leipzig: Georg Thieme.

Frey S, Campbell JS, Pike GB, Petrides M (2008) Dissociating the human language pathways with high angular resolution diffusion fiber tractography. J Neurosci 28:11435-11444.

Fukunaga M, Li TQ, van Gelderen P, de Zwart JA, Shmueli K, Yao B, Lee J, Maric D, Aronova MA, Zhang G, Leapman RD, Schenck JF, Merkle H, Duyn JH (2010) Layer-specific variation of iron content in cerebral cortex as a source of MRI contrast. Proc Natl Acad Sci U S A 107:3834-3839.

Gervain J, Macagno F, Cogoi S, Peña M, Mehler J (2008) The neonate brain detects speech structure. Proc Natl Acad Sci U S A 105:14222-14227.

Grossmann T, Johnson MH, Lloyd-Fox S, Blasi A, Deligianni F, Elwell C, Csibra G (2008) Early cortical specialization for face-to-face communication in human infants. Proc Biol Sci 275:2803-2811.

Hein G, Knight RT (2008) Superior temporal sulcus-it's my area: or is it? J Cogn Neurosci 20:2125-2136.

Hill J, Dierker D, Neil J, Inder T, Knutsen A, Harwell J, Coalson T, Van Essen D (2010) A surface-based analysis of hemispheric asymmetries and folding of cerebral cortex in term-born human infants. $J$ Neurosci 30:2268-2276.

Huttenlocher PR, Dabholkar AS (1997) Regional differences in synaptogenesis in human cerebral cortex. J Comp Neurol 387:167-178.

Johnson EK, Jusczyk PW (2001) Word segmentation by 8-month-olds: when speech cues count more than statistics. J Mem Lang 44:548-567. 
Kaas JH, Hackett TA (2000) Subdivisions of auditory cortex and processing streams in primates. Proc Natl Acad Sci U S A 97:11793-11799.

Kinney HC, Brody BA, Kloman AS, Gilles FH (1988) Sequence of central nervous system myelination in human infancy. II. Patterns of myelination in autopsied infants. J Neuropathol Exp Neurol 47:217-234.

Lerch JP, Worsley K, Shaw WP, Greenstein DK, Lenroot RK, Giedd J, Evans AC (2006) Mapping anatomical correlations across cerebral cortex (MACACC) using cortical thickness from MRI. Neuroimage 31:9931003.

Maye J, Werker JF, Gerken L (2002) Infant sensitivity to distributional information can affect phonetic discrimination. Cognition 82:B101-B111.

Ochiai T, Grimault S, Scavarda D, Roch G, Hori T, Rivière D, Mangin JF, Régis J (2004) Sulcal pattern and morphology of the superior temporal sulcus. Neuroimage 22:706-719.

Rilling JK, Glasser MF, Preuss TM, Ma X, Zhao T, Hu X, Behrens TE (2008) The evolution of the arcuate fasciculus revealed with comparative DTI. Nat Neurosci 11:426-428.

Rousseau F, Glenn OA, Iordanova B, Rodriguez-Carranza C, Vigneron DB, Barkovich JA, Studholme C (2006) Registration-based approach for reconstruction of high-resolution in utero fetal MR brain images. Acad Radiol 13:1072-1081.

Saffran JR, Aslin RN, Newport EL (1996) Statistical learning by 8-monthold infants. Science 274:1926-1928.

Scott SK, McGettigan C, Eisner F (2009) A little more conversation, a little less action-candidate roles for the motor cortex in speech perception. Nat Rev Neurosci 10:295-302.

Sigalovsky IS, Fischl B, Melcher JR (2006) Mapping an intrinsic MR property of gray matter in auditory cortex of living humans: a possible marker for primary cortex and hemispheric differences. Neuroimage 32: $1524-1537$.

Simonds RJ, Scheibel AB (1989) The postnatal development of the motor speech area: a preliminary study. Brain Lang 37:42-58.

Sowell ER, Peterson BS, Thompson PM, Welcome SE, Henkenius AL, Toga AW (2003) Mapping cortical change across the human life span. Nat Neurosci 6:309-315.

Sun T, Patoine C, Abu-Khalil A, Visvader J, Sum E, Cherry TJ, Orkin SH, Geschwind DH, Walsh CA (2005) Early asymmetry of gene transcription in embryonic human left and right cerebral cortex. Science 308:1794-1798.

Van Essen DC (2005) A population-average, landmark- and surface-based (PALS) atlas of human cerebral cortex. Neuroimage 28:635-662.

Vovk U, Pernus F, Likar B (2007) A review of methods for correction of intensity inhomogeneity in MRI. IEEE Trans Med Imaging 26:405-421.

Yakovlev P, Lecours AR (1967) The myelogenetic cycles of regional maturation of the brain. In: Regional development of the brain in early life (Minkovski A, ed), pp 3-69. Oxford: Blackwell.

Yoshiura T, Higano S, Rubio A, Shrier DA, Kwok WE, Iwanaga S, Numaguchi Y (2000) Heschl and superior temporal gyri: low signal intensity of the cortex on T2-weighted MR images of the normal brain. Radiology 214:217-221. 